\title{
Epistasis analysis of 16S rRNA ram mutations helps define the conformational dynamics of the ribosome that influence decoding
}

\author{
LANQING YING and KURT FREDRICK \\ Department of Microbiology and Center for RNA Biology, The Ohio State University, Columbus, Ohio 43210, USA
}

\begin{abstract}
The ribosome actively participates in decoding, with a tRNA-dependent rearrangement of the $30 \mathrm{~S}$ A site playing a key role. Ribosomal ambiguity (ram) mutations have mapped not only to the A site but also to the h12/S4/S5 region and intersubunit bridge B8, implicating other conformational changes such as $30 \mathrm{~S}$ shoulder rotation and B8 disruption in the mechanism of decoding. Recent crystallographic data have revealed that mutation G299A in helix h12 allosterically promotes B8 disruption, raising the question of whether G299A and/or other ram mutations act mainly via B8. Here, we compared the effects of each of several ram mutations in the absence and presence of mutation h8 $\Delta 2$, which effectively takes out bridge B8. The data obtained suggest that a subset of mutations including G299A act in part via B8 but predominantly through another mechanism. We also found that G299A in h12 and G347U in h14 each stabilize tRNA in the A site. Collectively, these data support a model in which rearrangement of the $30 \mathrm{~S}$ A site, inward shoulder rotation, and bridge B8 disruption are loosely coupled events, all of which promote progression along the productive pathway toward peptide bond formation.
\end{abstract}

Keywords: translation; EF-Tu; aminoacyl-tRNA selection; codon-anticodon; fidelity

\section{INTRODUCTION}

During the decoding step of protein synthesis, aminoacyltRNA (aa-tRNA) binds the ribosome as part of a ternary complex with EF-Tu and GTP (for review, see Rodnina 2012). Initial binding of EF-Tu•GTP•aa-tRNA is followed by sampling of codon-anticodon interactions in the $30 \mathrm{~S} \mathrm{~A}$ site. Pairing between codon and anticodon leads to GTPase activation and GTP hydrolysis, which allows release of aatRNA from EF-Tu. The acceptor end of aa-tRNA then either moves completely into the A site (a step termed "accommodation"), where it can participate in peptidyl transfer, or is rejected from the ribosome.

The accuracy of decoding is quite high, with measured error rates in bacterial cells on the order of $10^{-3}-10^{-5}$, depending on the nature of the mismatch (Kramer and Farabaugh 2007; Manickam et al. 2014). This level of accuracy relies in part on a kinetic proofreading mechanism (Hopfield 1974; Ninio 1975; Ruusala et al. 1982). In essence, differences in the energetics of codon-anticodon pairing are exploited twice, before and after the functionally irreversible GTP hydrolysis step, providing two independent opportunities for incorrect aa-tRNA rejection. Matched (cognate) codon-anti-

Corresponding author: fredrick.5@osu.edu

Article published online ahead of print. Article and publication date are at http://www.rnajournal.org/cgi/doi/10.1261/rna.054486.115. codon pairing not only stabilizes aa-tRNA but also increases the rate of GTPase activation/GTP hydrolysis and accommodation (Pape et al. 1999; Gromadski and Rodnina 2004; Geggier et al. 2010). This indicates that discrimination entails induced fit or conformational selection at both the initial selection and proofreading stages of decoding.

There is considerable evidence that the ribosome plays an active role in decoding. Structural studies have shown that, upon A-site tRNA (A-tRNA) binding, universally conserved nucleotides A1492, A1493, and G530 of the 16S rRNA rearrange to dock against the codon-anticodon helix (Ogle et al. 2001; Selmer et al. 2006; Schmeing et al. 2009; Voorhees et al. 2010; Demeshkina et al. 2012). This A-site rearrangement excludes solvent from the minor groove of the codon-anticodon helix. Consequently, a mismatch between codon and anticodon (particularly at the first or second position) makes the rearrangement highly unfavorable, due to uncompensated $\mathrm{H}$-bond donor/acceptor groups. Indeed, differences in the thermodynamics of this A-site rearrangement may be fundamentally responsible for substrate discrimination at both stages of decoding (Satpati et al. 2014).

(C) 2016 Ying and Fredrick This article is distributed exclusively by the RNA Society for the first 12 months after the full-issue publication date (see http:// rnajournal.cshlp.org/site/misc/terms.xhtml). After 12 months, it is available under a Creative Commons License (Attribution-NonCommercial 4.0 International), as described at http://creativecommons.org/licenses/by-nc/4.0/. 
While the A-site rearrangement is undoubtedly a key aspect of GTPase activation, how these changes in the $30 \mathrm{~S} \mathrm{~A}$ site promote GTP hydrolysis $\sim 80 \AA$ away remains unclear. Structures of the ternary complex bound to the ribosome show that the tRNA adopts a distorted A/T conformation, involving a $\sim 30^{\circ}$ bend in the anticodon arm and substantial alterations in the elbow and acceptor-end regions (Schmeing et al. 2009; Voorhees et al. 2010). Changes in ribosome conformation have also been seen upon A-tRNA binding, including $30 \mathrm{~S}$ head domain movement and inward $30 \mathrm{~S}$ shoulder domain rotation, changes collectively referred to as "domain closure" (Ogle et al. 2001, 2002). These conformational changes in the ribosome and the ternary complex may jointly contribute to GTPase activation and functionally depend on the A-site rearrangement.

A number of mutations that alter the fidelity of decoding have been identified in the ribosome, confirming its importance in the process. Most ribosomal ambiguity ( $\mathrm{ram}$ ) mutations are believed to generally promote aminoacyl-tRNA incorporation (like high concentrations of $\mathrm{Mg}^{2+}$ ) and thereby reduce the accuracy of aa-tRNA selection (Johansson et al. 2012; McClory et al. 2014; Zhang et al. 2015). Ram mutations have mapped not only to the A site but also to distal regions (Fig. 1). These include the h12/S4/S5 region, which lies on the solvent side of the subunit at an interface between the shoulder subdomain and the remainder of the subunit; h16, which represents the "top" of the shoulder; and h8/h14/L19; elements which contribute to intersubunit bridge B8 (Maisnier-Patin et al. 2002, 2007; McClory et al. 2010). A recent reevaluation of S4/S5 mutations showed that all C-terminal truncations of $\mathrm{S} 4$ confer a ram phenotype (Agarwal et al. 2015). These truncations undoubtedly destabilize the open conformation of the subunit, lending strong support to the idea that domain closure contributes to GTPase activation (Ogle and Ramakrishnan 2005; Voorhees et al. 2010; Fredrick 2015). Ram mutations in h8/h14 act by disrupting bridge B8. Truncation of either helix confers a strong ram phenotype and accelerates GTPase activation in vitro, indicating that the B8 normally contributes to the energetic barrier of this step (McClory et al. 2010). Indeed, X-ray crystallography studies have shown that either ram mutations or the binding of EF-Tu•GDPCP•aa-tRNA causes disruption of B8, suggesting that GTPase activation normally entails the physical disruption of B8 (Schmeing et al. 2009; Voorhees et al. 2010; Fagan et al. 2013).

Further studies of a representative subset of $16 \mathrm{~S}$ rRNA ram mutations have shown that they confer defects in both the initial selection and proofreading phases of decoding (McClory et al. 2014). Mutations in h8/h14 (h8 $\Delta 3$, h14 $\Delta 2$, G347U), h12 (G299A), h34 (C1054A, C1054U, C1200U), and h44 (G1491A) similarly increase $k_{\text {cat }}$ of GTP hydrolysis in single-turnover reactions by approximately twofold and approximately ninefold for cognate and near-cognate ternary complexes, respectively, with small (approximately twofold) increases in $K_{M}$ in the cognate case (McClory et al. 2010, 2014; Fagan et al. 2013). These effects, interpreted in light of earlier work (Pape et al. 1998, 1999; Gromadski and Rodnina 2004), indicate that the mutations accelerate the GTPase activation step of initial selection (McClory et al. 2010). These same mutations were also found to cause defects in the proofreading phase, reducing the rejection rate of near-cognate aa-tRNA after GTP hydrolysis typically by three- to eightfold (McClory et al. 2014). The A-site mutations $\mathrm{C} 1054 \mathrm{U}$ and $\mathrm{C} 1054 \mathrm{~A}$ were somewhat exceptional in that they altered proofreading in a tRNA-specific way. Nonetheless, these findings suggest that most ram mutations promote aa-tRNA incorporation in general at both stages of decoding and thereby reduce the accuracy of the process. While these various ram mutations similarly promote aatRNA incorporation, the mechanism(s) by which they act remain ambiguous.

In the crystal structure of ribosomes carrying mutation G299A of h12, bridge B8 was seen to be disrupted (Fagan et al. 2013), suggesting that G299A acts, at least in part, by
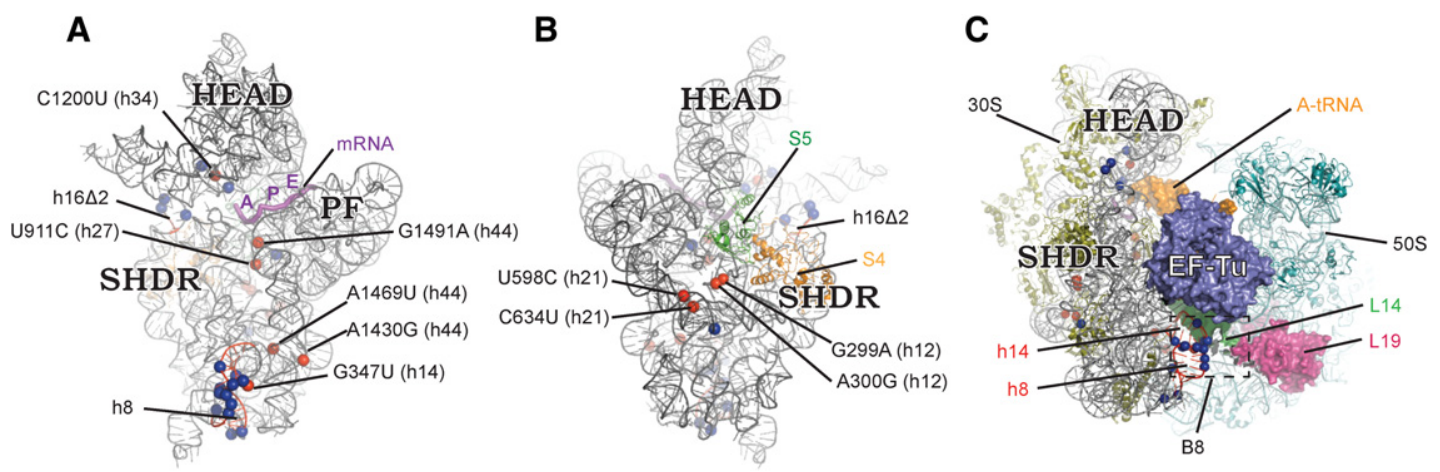

FIGURE 1. Locations of ram mutations. Positions of mutations that increase miscoding are shown on the tertiary structure of $16 \mathrm{~S}$ rRNA, viewed from the interface $(A)$ and solvent $(B)$ perspective, and in the context of the complete $70 S$ ribosome with bound ternary complex $(C)$. Red spheres indicate mutations studied in this work; blue spheres indicate other $16 \mathrm{~S} \mathrm{ram} \mathrm{mutations.} \mathrm{Helices} \mathrm{h8} \mathrm{and} \mathrm{h14} \mathrm{are} \mathrm{highlighted} \mathrm{in} \mathrm{red,} \mathrm{as} \mathrm{is} \mathrm{the} \mathrm{portion} \mathrm{of} \mathrm{h16}$ removed by deletion. With the exception of S4 and S5 (as indicated), ribosomal proteins are computationally omitted in panels $A$ and $B$ to better reveal the rRNA. SHDR, 30 S shoulder. PF, platform. Images were made in PyMOL using PDB entries 2WRN and 2WRO. 
allosterically destabilizing B8. Here, we use genetic epistasis to evaluate the degree to which the effects of G299A and various other $16 \mathrm{~S}$ ram mutations depend on B8. The results suggest that G299A and several other mutations act in part via B8 but primarily through another mechanism. We also show that G299A and G347U stabilize A-tRNA, indicating that changes in h12 and h14 allosterically influence the 30S A site. These observations shed light on the conformational dynamics of the subunit that impact the decoding process.

\section{RESULTS AND DISCUSSION}

\section{Genetic epistasis analysis of $16 \mathrm{~S}$ ram mutations}

To investigate the relationship between bridge $\mathrm{B} 8$ and the mechanism by which various ram mutations act, each was combined with mutation $\mathrm{h} 8 \Delta 2$, and the resulting double mutant ribosomes were assayed for miscoding in vivo. Mutation $\mathrm{h} 8 \Delta 2$ corresponds to a 2 -bp truncation of $\mathrm{h} 8$, predicted to disrupt the h8/h14 tertiary interaction and hence B8 (Fig. 1; McClory et al. 2010). The basic rationale here is that, because $\mathrm{h} 8 \Delta 2$ "predisrupts" $\mathrm{B} 8$, any mutation that normally acts via $\mathrm{B} 8$ will be unable (or less able) to increase miscoding in the presence of h8 2 (Carter et al. 1984; Wells 1990; LiCata and Ackers 1995). We first quantified GAU misreading by Glu-tRNA (Fig. 2A). As expected, moving h8 82 into ribosomes containing a 2-bp truncation of h14 (h14 $\Delta 2$ ) did not further increase miscoding. Similar positive epistasis was seen between $\mathrm{h} 8 \Delta 2$ and G347U, a point mutation that has been observed in crystallographic studies to disrupt B8 (McClory et al. 2010; Fagan et al. 2013). These data confirm that $\mathrm{h} 8 \Delta 2$, h14 $\Delta 2$, and G347U all act via the same mechanism - destabilization of B8. In contrast, when h8 82 was combined with each of the other ram mutations analyzed, substantial increases in miscoding were seen (Fig. 2A). For mutations C1200U and h16 2 2, fold-effects were found to be multiplicative in the context of the double mutant (i.e., no epistasis), suggesting that these mutations act through a mechanism independent of B8. Effects of G1491A and h8 82 in combination were slightly more than multiplicative (i.e., negative epistasis), implying some degree of synergism. For the other mutations (G299A, A300G, U598C, C634U, U911C, A1430G, and C1469U), fold-effects were somewhat less than multiplicative in the context of the double mutant, suggesting that these mutations act in part through B8 but primarily via another mechanism.

We next compared these mutant ribosomes using a nonsense suppression assay, which quantifies misreading of UGA, presumably by Trp-tRNA (Fig. 2B). The data obtained were generally consistent with those from the missense suppression assay. Again, strong positive epistasis was seen for $\mathrm{h} 8 \Delta 2 / \mathrm{h} 14 \Delta 2$ and h8 $82 / \mathrm{G} 347 \mathrm{U}$ combinations, as expected.
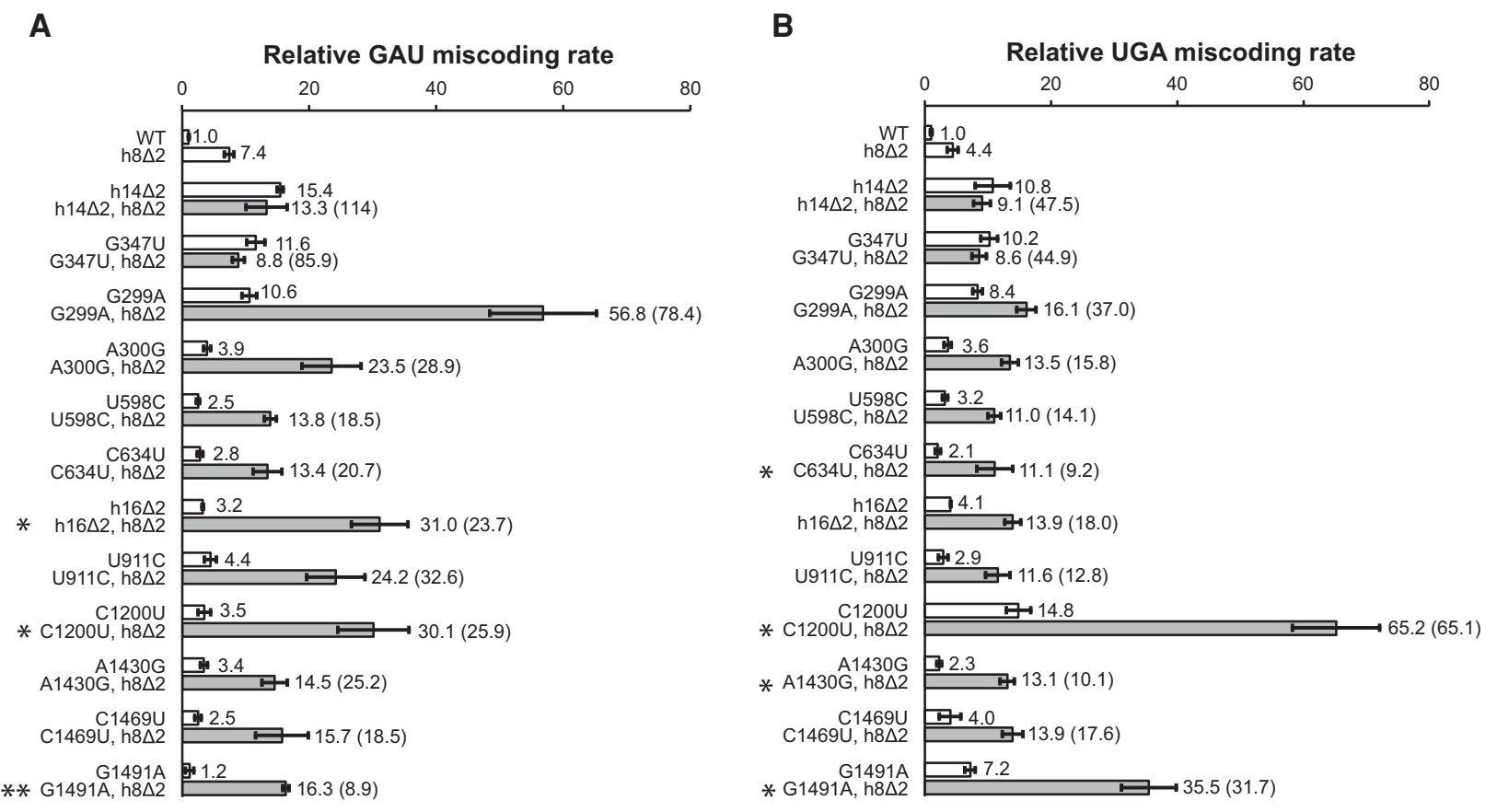

FIGURE 2. Genetic epistasis analysis of $16 \mathrm{~S}$ ram mutations. Shown are the relative rates of GAU $(A)$ and UGA $(B)$ misreading by control and mutant ribosomes, as indicated. Control (WT) and single mutants, white bars; double mutants, gray bars. A-site mutation C1054A could not be included in this analysis because ribosomes containing h8 82 and C1054A exhibited very low translation activity. Data correspond to the mean \pm SEM from three or more independent experiments. Under these conditions, the control (WT) ribosomes exhibit absolute frequencies of miscoding of GAU and UGA of 0.0011 and 0.0058 , respectively. Values in parentheses correspond to the product of the fold-effects of the two relevant single mutations. Cases where miscoding rate in the double mutant is as high as this product are scored as no epistasis $\left({ }^{*}\right)$ or negative epistasis $\left({ }^{* *}\right)$. 
No epistasis was seen between h8 22 and C634U, C1200U, A1430G, or G1491A, suggesting that these mutations increase UGA miscoding via a mechanism completely independent of B8. The remainder of mutations appeared to exhibit some degree of positive epistasis with h8 2 , with G299A being the clearest case. However, the fold-increase in UGA miscoding seen for many of these double-mutant ribosomes nearly approached the product of fold-increases of the individual mutations, suggesting that $\mathrm{B} 8$ has little bearing on their mechanisms of action.

While generally congruent, the missense and nonsense suppression results showed some differences. Namely, the latter data indicate a larger degree of positive epistasis between h8 22 and G299A, and no epistasis between h8 $\Delta 2$ and C634U, A1430G, or G1491A. These differences might stem from effects of RF2 activity, which would be expected to impact the UGA read-through assay in particular. An alternative (and non-mutually-exclusive) possibility is this variation is related to the distinct aa-tRNA species involved in the miscoding events.

As noted previously (McClory et al. 2010), the single mutations C1200U and G1491A have a much larger effect on UGA read-through than on GAU miscoding, which cannot be readily attributed to altered RF2 activity (McClory et al. 2014). These mutations in or near the $\mathrm{A}$ site are unique in that they increase miscoding in vitro in a context-dependent manner, causing high error rates for only particular aa-tRNAs and/or codons (McClory et al. 2014). Interestingly, C1200U and G1491A are the two mutations analyzed here that consistently show no positive epistasis with h8 $\Delta 2$ (Fig. 2).

\section{Mutations G299A and G347U stabilize A-site tRNA}

It has been proposed that rearrangement of the A site induced by tRNA binding is coupled to more global changes in the subunit structure, such as inward rotation of the $30 \mathrm{~S}$ shoulder domain (Ogle and Ramakrishnan 2005). If this is indeed the case, ribosomal mutations that promote such conforma- tional changes should stabilize A-tRNA binding, based on the principle of microscopic reversibility. To investigate this, we used a filter-binding assay to measure the affinity of cognate tRNA for the A site of control and mutant ribosomes. Two species of tRNA were used, $\mathrm{tRNA}^{\text {Glu }}$ and $\mathrm{tRNA}^{\text {Phe }}$, and comparable results were seen in both cases (Fig. 3). Mutation G299A and G347U each significantly increased the affinity of tRNA for the A site, reducing the $K_{\mathrm{D}}$ by four- to sixfold and two- to threefold, respectively. These data provide evidence that A-tRNA binding is conformationally coupled to both h12 and h14, with each mutation stabilizing A-tRNA. An important point to emphasize here is that these ram mutations stabilize A-tRNA in the absence of EF-Tu. Thus, their ability to promote aa-tRNA incorporation during decoding probably stems from their effects on the intrinsic dynamics of the ribosome and A-tRNA affinity, as opposed to effects on EF-Tu binding.

\section{Conformational dynamics of the $30 \mathrm{~S}$ subunit that impact decoding}

We envisage that, as proposed previously (Satpati et al. 2014), the ribosome adopts two basic states functionally relevant to decoding: open (off) and closed (on). Genetic and structural evidence suggest that formation of the closed (on) state involves not only rearrangement of the A site but also conformational changes elsewhere, such as disruption of bridge B8 and inward rotation of the 30S shoulder (Ogle and Ramakrishnan 2005; McClory et al. 2010; Fagan et al. 2013; Fredrick 2015). Binding of tRNA to the $30 \mathrm{~S}$ A site promotes the open-to-closed transition, and vice versa, as depicted in the thermodynamic cycles of Figure 4. Mutations G299A and $\mathrm{G} 347 \mathrm{U}$ each shift the equilibria rightward (i.e., increase both $K_{C}$ and $K_{C^{\prime}}$ by the same factor), stabilizing the closed (on) state and hence A-site tRNA as well (Fig. 4B). As formation of the closed (on) state is a prerequisite for GTPase activation (Satpati et al. 2014), the ability of these ram mutations to promote the open-to-closed transition explains how they
A

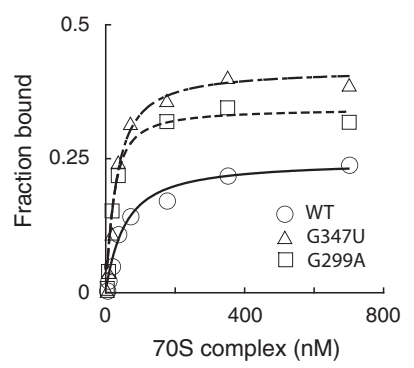

B

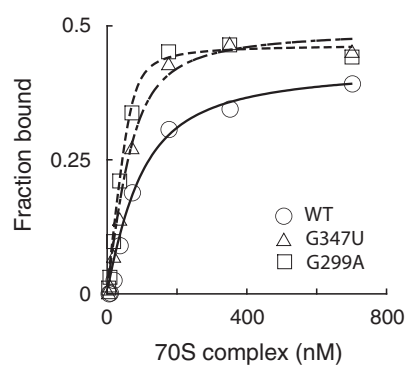

C

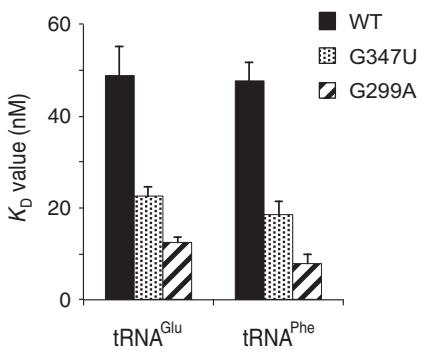

FIGURE 3. Effects of $16 \mathrm{~S}$ rRNA mutations G347U and G299A on the binding of cognate tRNA to the A site. Shown are examples of equilibrium binding experiments, in which ribosomes (O, WT control; $\Delta, \mathrm{G} 347 \mathrm{U} ; \square, \mathrm{G} 299 \mathrm{~A})$ containing tRNA ${ }^{\mathrm{fMet}}$ in the $\mathrm{P}$ site and a cognate codon in the A site were incubated (at various concentrations) with $\left[3^{\prime}-{ }^{32} \mathrm{P}\right]-\mathrm{tRNA}^{\mathrm{Glu}}(A)$ or $\left[3^{\prime}-{ }^{32} \mathrm{P}\right]-\mathrm{tRNA}^{\mathrm{Phe}}(B)$, and the fraction of bound A-tRNA determined by filtration through a membrane bilayer. Data were fit to a quadratic function to obtain the $K_{\mathrm{D}}$ values shown in panel $C$, each representing the mean \pm SEM from three or more independent experiments. WT, black bars; G347U, dotted bars; G299A, striped bars. 

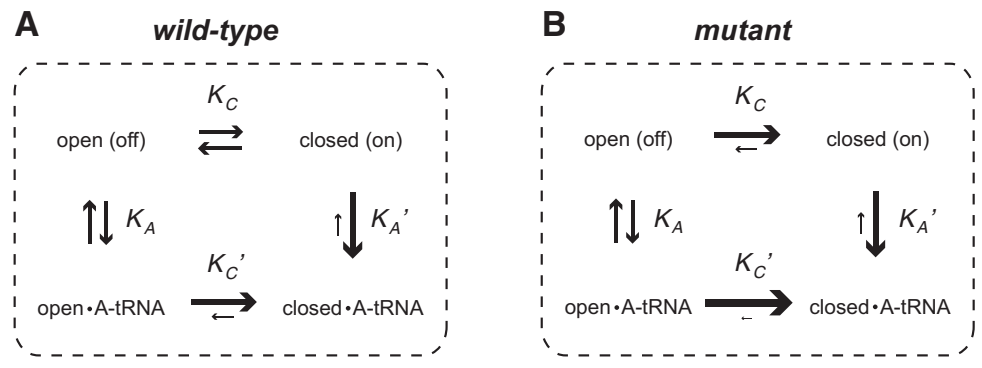

FIGURE 4. A simplified model in which the ribosome adopts two distinct conformations pertinent to decoding. Shown are thermodynamic cycles ( $A$, wild-type; $B$, mutant) in which A-tRNA binding promotes transition from the open (off) to closed (on) conformational state of the ribosome and vice versa. $K_{C}$ and $K_{C^{\prime}}$ represent the equilibrium constants for the open-to-closed transition in the absence and presence of A-tRNA, respectively; $K_{A}$ and $K_{A^{\prime}}$ represent the equilibrium association constants for A-tRNA binding to the open and closed ribosome, respectively. In each case $(A, B), K_{C} K_{A^{\prime}}=K_{A} K_{C}$. In the wild-type case $(A)$, the equilibrium constants $K_{A}$ and $K_{C}$ are arbitrarily set to 1 (i.e., forward and reverse arrows are identically sized) and serve as reference points for the entire figure. Differences in arrow sizes signify relative differences in equilibria. (Arrow sizes should not be taken as direct reflections of forward or reverse rate constants.) We propose that the open-to-closed transition entails rearrangement of A-site nucleotides (A1492, A1493, G530), inward rotation of the 30S shoulder, and disruption of B8. Hence this scheme is oversimplified in that these multiple partially coupled events are shown grouped into a single step. Mutations such as G299A and G347U shift the equilibria rightward (B), increasing $K_{C}$ and $K_{C}$ by the same factor, and hence promoting formation of the closed•A-tRNA state. While G299A and G347U each promote the open-to-closed transition, they do so in largely independent waysfor example by promoting shoulder movement and weakening B8, respectively.

accelerate GTPase activation and reduce the stringency of initial selection (McClory et al. 2010; Fagan et al. 2013). Furthermore, the ability of G299A and G347U to stabilize tRNA in the A site explains in a simple way how they can compromise proofreading (McClory et al. 2014).

Our epistasis analysis suggests that G299A and G347U promote the open-to-closed transition via largely independent mechanisms. Ribosomes with G347U show a frequency of miscoding that is unenhanced by the additional presence of h8 82 (Fig. 2), providing evidence that both G347U and h8 82 act via the same mechanism-destabilization of B8. On the other hand, the frequency of miscoding in G299A ribosomes is increased substantially by the additional presence of h8 82 , indicating that G299A acts mainly through another mechanism. One possibility is that G299A promotes inward shoulder rotation, as has been suggested for nearby mutations at the S4/S5 interface (Ogle and Ramakrishnan 2005). Consistent with this idea, nucleotide G299 is normally involved in the coordination of a magnesium ion, and loss of this $\mathrm{Mg}^{2+}$ (as is seen in G299A ribosomes) may indeed alter 30 S shoulder dynamics (McClory et al. 2010; Fagan et al. 2013).

Arguably, biophysical studies of the pretranslocation (PRE) complex represent the most comprehensive look at ribosome dynamics to date (Fei et al. 2008, 2011; Fischer et al. 2010; Munro et al. 2010; Agirrezabala et al. 2012). The PRE complex is inherently dynamic, with reversible intersubunit rotation, head swiveling, and L1 stalk movements, along with fluctuations of tRNAs between classic and hybrid configurations. These motions (e.g., P/P to P/E movement, counterclockwise
30 Sody rotation and head swiveling, and inward L1 movement) are loosely coupled to one another, such that two global states predominate in the overall conformational landscape. These observations shape our view of ribosome dynamics in general, and lead us to propose that motions important for decoding (rearrangement of the A site, inward rotation of the shoulder, and disruption of B8) are loosely coupled as well. This is consistent with the small degree of positive epistasis observed between $\mathrm{h} 8 \Delta 2$ and mutations predicted to promote shoulder rotation (e.g., G299A, A300G) (Fig. 2), earlier crystallographic evidence that G299A promotes B8 disruption (Fagan et al. 2013), and the enhancement of A-tRNA binding caused by G347U and G299A (Fig. 3).

Finally, our findings here underscore the importance of ribosome dynamics in the decoding process. Mutations in ribosomal elements that lie $\sim 80 \AA$ away from the $30 \mathrm{~S}$ A site can together reduce the accuracy of decoding by more than 50 -fold. Interestingly, about one-third of the ram mutations isolated in the Esherichia coli $16 \mathrm{~S}$ rRNA map to nonconserved nucleotides, with the ram-conferring substitutions being well represented in nature (Cannone et al. 2002; McClory et al. 2010). Natural variation at these positions may in part reflect the evolutionary tuning of decoding fidelity across different lineages. Indeed, it seems likely that the ribosome has evolved in each organism to strike the optimal balance between translation speed and accuracy in the context of its particular intracellular milieu.

\section{MATERIALS AND METHODS}

\section{Measurements of miscoding in vivo}

Rates of miscoding were measured using an orthologous translation system as described previously (Abdi and Fredrick 2005; McClory et al. 2010, 2011). Briefly, three indicator strains were usedKLF4001, KLF2723, and KLF2674, each carrying the lacZ reporter in single copy on the chromosome. In these strains, lac $Z$ lies downstream from the consensus $\mathrm{P}_{\text {ant }}$ promoter and is preceded by the alternative Shine-Dalgarno sequence $5^{\prime}$-ATCCC-3' $\left(\mathrm{SD}^{*}\right)$. In KLF4001, lacZ carries a missense mutation at codon 461 (GAA to GAT); in KLF2723, lacZ contains nonsense mutation at codon 585 (TGG to TGA); in KLF2674, lacZ contains no mutations (control strain). Plasmid pKF207 encodes $16 \mathrm{~S}$ rRNA with the complementary (orthologous) anti-SD sequence $5^{\prime}$-GGGGU-5' (ASD*). Variants of this plasmid with one or two additional mutations in the 16S rRNA gene (single or double mutants) were transformed into each of the indicator strains, and the activity of $\beta$-galactosidase 
in the resulting transformants was determined. For each of the plasmid-encoded orthologous ribosomes, rates of miscoding were calculated as the level of active $\beta$-galactosidase produced from the missense or nonsense reporter divided by that from the control reporter (McClory et al. 2010).

\section{Biochemical reagents}

Purified tRNA ${ }^{\text {Glu }}$ and tRNA ${ }^{\text {Phe }}$ were purchased from Chemical Block (Moscow, Russia) and radiolabeled as described previously (McGarry et al. 2005). Ribosomes were purified from E. coli $\Delta 7$ prrn strains, and model mRNAs derived from gene 32 of phage T4 were prepared as described previously (Fredrick and Noller 2002; Qin et al. 2007; Liu and Fredrick 2013).

\section{tRNA binding experiments}

The affinity of tRNA for the A site was measured using a doublemembrane filtration method (Wong and Lohman 1993; Fahlman and Uhlenbeck 2004; Walker and Fredrick 2006). In the case of tRNA $^{\text {Glu }}$, for example, ribosomes $(0.7 \mu \mathrm{M})$ were first incubated at $37^{\circ} \mathrm{C}$ for $20 \mathrm{~min}$ in polymix buffer $(5 \mathrm{mM}$ potassium phosphate $\mathrm{pH}$ 7.3, $95 \mathrm{mM} \mathrm{KCl}, 5 \mathrm{mM} \mathrm{NH}_{4} \mathrm{Cl}, 0.5 \mathrm{mM} \mathrm{CaCl}_{2}, 5 \mathrm{mM}$ magnesium acetate, $8 \mathrm{mM}$ putrescine, $1 \mathrm{mM}$ spermidine, $1 \mathrm{mM} \mathrm{DTT}$ ) with tRNA $^{\text {fMet }}(0.7 \mu \mathrm{M})$ and $\mathrm{m} 640(1.5 \mu \mathrm{M})$, a model mRNA that has GAA as codon 2 (Shoji et al. 2009). The resulting complex, diluted to various concentrations $(3.5-700 \mathrm{nM})$, was further incubated for 40 min with $\left[3^{\prime}-{ }^{32} \mathrm{P}\right]$-tRNA ${ }^{\text {Glu }}(<0.1 \mu \mathrm{M})$. Reactions were then filtered through a bilayer of nitrocellulose and nylon membranes (NitroBind and Hybond- $\mathrm{N}^{+}$; GE Healthcare), and the membranes were washed immediately with $150 \mu \mathrm{L}$ polymix buffer, dried, and subjected to phosphor imager analysis. The fraction of tRNA bound was quantified and plotted as a function of ribosome concentration. Data were fit to the equation $F=F_{\max }\left\{\left(A+B+K_{\mathrm{D}}\right)-[(A+B+\right.$ $\left.\left.\left.K_{\mathrm{D}}\right)^{2}-4 A B\right]^{1 / 2}\right\} /(2 A)$, where $F$ represents the fraction of tRNA bound, $A$ represents the input concentration of radiolabeled tRNA, and $B$ represents the input concentration of programmed ribosomes, yielding the apparent dissociation constant $\left(K_{\mathrm{D}}\right)$ and maximal $F$ value $\left(F_{\max }\right)$ for each experiment. For tRNA ${ }^{\text {Phe }}$ binding, experiments were performed in the same way, except that message m291, in which UUU is codon 2 (Shoji et al. 2009), was used.

\section{ACKNOWLEDGMENTS}

We thank A. Hinnebusch for suggesting this line of investigation, J. Jackman for providing ATP(CTP):tRNA nucleotidyltransferase, and M. Ibba for comments on the manuscript. This work was supported by National Institutes of Health grant no. GM072528.

Received September 15, 2015; accepted January 14, 2016.

\section{REFERENCES}

Abdi NM, Fredrick K. 2005. Contribution of 16S rRNA nucleotides forming the 30 S subunit A and P sites to translation in Escherichia coli. RNA 11: 1624-1632.

Agarwal D, Kamath D, Gregory ST, O'Connor M. 2015. Modulation of decoding fidelity by ribosomal proteins S4 and S5. J Bacteriol 197: 1017-1025.
Agirrezabala X, Liao HY, Schreiner E, Fu J, Ortiz-Meoz RF, Schulten K, Green R, Frank J. 2012. Structural characterization of mRNA-tRNA translocation intermediates. Proc Natl Acad Sci 109: 6094-6099.

Cannone JJ, Subramanian S, Schnare MN, Collett JR, D'Souza LM, Du Y, Feng B, Lin N, Madabusi LV, Müller KM, et al. 2002. The comparative RNA web (CRW) site: an online database of comparative sequence and structure information for ribosomal, intron, and other RNAs. BMC Bioinformatics 3: 2.

Carter PJ, Winter G, Wilkinson AJ, Fersht AR. 1984. The use of double mutants to detect structural changes in the active site of the tyrosyltRNA synthetase (Bacillus stearothermophilus). Cell 38: 835-840.

Demeshkina N, Jenner L, Westhof E, Yusupov M, Yusupova G. 2012. A new understanding of the decoding principle on the ribosome. Nature 484: 256-259.

Fagan CE, Dunkle JA, Maehigashi T, Dang MN, Devaraj A, Miles SJ, Qin D, Fredrick K, Dunham CM. 2013. Reorganization of an intersubunit bridge induced by disparate $16 \mathrm{~S}$ ribosomal ambiguity mutations mimics an EF-Tu-bound state. Proc Natl Acad Sci 110: 9716-9721.

Fahlman RP, Uhlenbeck OC. 2004. Contribution of the esterified amino acid to the binding of aminoacylated tRNAs to the ribosomal P- and A-sites. Biochemistry 43: 7575-7583.

Fei J, Kosuri P, MacDougall DD, Gonzalez RL Jr. 2008. Coupling of ribosomal L1 stalk and tRNA dynamics during translation elongation. Mol Cell 30: 348-359.

Fei J, Richard AC, Bronson JE, Gonzalez RL Jr. 2011. Transfer RNA-mediated regulation of ribosome dynamics during protein synthesis. Nat Struct Mol Biol 18: 1043-1051.

Fischer N, Konevega AL, Wintermeyer W, Rodnina MV, Stark H. 2010. Ribosome dynamics and tRNA movement by time-resolved electron cryomicroscopy. Nature 466: 329-333.

Fredrick K. 2015. Another look at mutations in ribosomal protein S4 lends strong support to the domain closure model. J Bacteriol 197: 1014-1016.

Fredrick K, Noller HF. 2002. Accurate translocation of mRNA by the ribosome requires a peptidyl group or its analog on the tRNA moving into the 30S P site. Mol Cell 9: 1125-1131.

Geggier P, Dave R, Feldman MB, Terry DS, Altman RB, Munro JB, Blanchard SC. 2010. Conformational sampling of aminoacyl-tRNA during selection on the bacterial ribosome. J Mol Biol 399: 576-595.

Gromadski KB, Rodnina MV. 2004. Kinetic determinants of high-fidelity tRNA discrimination on the ribosome. Mol Cell 13: 191-200.

Hopfield JJ. 1974. Kinetic proofreading: a new mechanism for reducing errors in biosynthetic processes requiring high specificity. Proc Natl Acad Sci 71: 4135-4139.

Johansson M, Zhang J, Ehrenberg M. 2012. Genetic code translation displays a linear trade-off between efficiency and accuracy of tRNA selection. Proc Natl Acad Sci 109: 131-136.

Kramer EB, Farabaugh PJ. 2007. The frequency of translational misreading errors in $E$. coli is largely determined by tRNA competition. RNA 13: 87-96.

LiCata VJ, Ackers GK. 1995. Long-range, small magnitude nonadditivity of mutational effects in proteins. Biochemistry 34: 3133-3139.

Liu Q, Fredrick K. 2013. Contribution of intersubunit bridges to the energy barrier of ribosomal translocation. Nucleic Acids Res 41: 565-574.

Maisnier-Patin S, Berg OG, Liljas L, Andersson DI. 2002. Compensatory adaptation to the deleterious effect of antibiotic resistance in Salmonella typhimurium. Mol Microbiol 46: 355-366.

Maisnier-Patin S, Paulander W, Pennhag A, Andersson DI. 2007. Compensatory evolution reveals functional interactions between ribosomal proteins S12, L14 and L19. J Mol Biol 366: 207-215.

Manickam N, Nag N, Abbasi A, Patel K, Farabaugh PJ. 2014. Studies of translational misreading in vivo show that the ribosome very efficiently discriminates against most potential errors. RNA 20: 9-15.

McClory SP, Leisring JM, Qin D, Fredrick K. 2010. Missense suppressor mutations in $16 \mathrm{~S}$ rRNA reveal the importance of helices h8 and h14 in aminoacyl-tRNA selection. RNA 16: 1925-1934. 
McClory SP, Devaraj A, Qin D, Leisring JM, Fredrick K. 2011. Mutations in $16 \mathrm{~S}$ rRNA that decrease the fidelity of translation. Ribosomes 237-247.

McClory SP, Devaraj A, Fredrick K. 2014. Distinct functional classes of ram mutations in 16S rRNA. RNA 20: 496-504.

McGarry KG, Walker SE, Wang H, Fredrick K. 2005. Destabilization of the $\mathrm{P}$ site codon-anticodon helix results from movement of tRNA into the P/E hybrid state within the ribosome. Mol Cell 20: 613-622.

Munro JB, Altman RB, Tung CS, Cate JH, Sanbonmatsu KY, Blanchard SC. 2010. Spontaneous formation of the unlocked state of the ribosome is a multistep process. Proc Natl Acad Sci 107: 709-714.

Ninio J. 1975. Kinetic amplification of enzyme discrimination. Biochimie 57: 587-595.

Ogle JM, Ramakrishnan V. 2005. Structural insights into translational fidelity. Annu Rev Biochem 74: 129-177.

Ogle JM, Brodersen DE, Clemons WM Jr, Tarry MJ, Carter AP, Ramakrishnan V. 2001. Recognition of cognate transfer RNA by the 30S ribosomal subunit. Science 292: 897-902.

Ogle JM, Murphy FV, Tarry MJ, Ramakrishnan V. 2002. Selection of tRNA by the ribosome requires a transition from an open to a closed form. Cell 111: 721-732.

Pape T, Wintermeyer W, Rodnina MV. 1998. Complete kinetic mechanism of elongation factor Tu-dependent binding of aminoacyltRNA to the A site of the E. coli ribosome. EMBO J 17: 7490-7497.

Pape T, Wintermeyer W, Rodnina M. 1999. Induced fit in initial selection and proofreading of aminoacyl-tRNA on the ribosome. EMBO J 18: $3800-3807$.

Qin D, Abdi NM, Fredrick K. 2007. Characterization of 16S rRNA mutations that decrease the fidelity of translation initiation. RNA 13: 2348-2355.
Rodnina MV. 2012. Quality control of mRNA decoding on the bacterial ribosome. Adv Protein Chem Struct Biol 86: 95-128.

Ruusala T, Ehrenberg M, Kurland CG. 1982. Is there proofreading during polypeptide synthesis? EMBO J 1: 741-745.

Satpati P, Sund J, Aqvist J. 2014. Structure-based energetics of mRNA decoding on the ribosome. Biochemistry 53: 1714-1722.

Schmeing TM, Voorhees RM, Kelley AC, Gao YG, Murphy FV IV, Weir JR, Ramakrishnan V. 2009. The crystal structure of the ribosome bound to EF-Tu and aminoacyl-tRNA. Science 326: 688-694.

Selmer M, Dunham CM, Murphy FV IV, Weixlbaumer A, Petry S, Kelley AC, Weir JR, Ramakrishnan V. 2006. Structure of the 70S ribosome complexed with mRNA and tRNA. Science 313: 1935-1942.

Shoji S, Abdi NM, Bundschuh R, Fredrick K. 2009. Contribution of ribosomal residues to P-site tRNA binding. Nucleic Acids Res 37: 4033-4042.

Voorhees RM, Schmeing TM, Kelley AC, Ramakrishnan V. 2010. The mechanism for activation of GTP hydrolysis on the ribosome. Science 330: 835-838.

Walker SE, Fredrick K. 2006. Recognition and positioning of mRNA in the ribosome by tRNAs with expanded anticodons. J Mol Biol 360: 599-609.

Wells JA. 1990. Additivity of mutational effects in proteins. Biochemistry 29: 8509-8517.

Wong I, Lohman TM. 1993. A double-filter method for nitrocellulosefilter binding: application to protein-nucleic acid interactions. Proc Natl Acad Sci 90: 5428-5432.

Zhang J, Ieong KW, Johansson M, Ehrenberg M. 2015. Accuracy of initial codon selection by aminoacyl-tRNAs on the mRNA-programmed bacterial ribosome. Proc Natl Acad Sci 112: 9602-9607. 

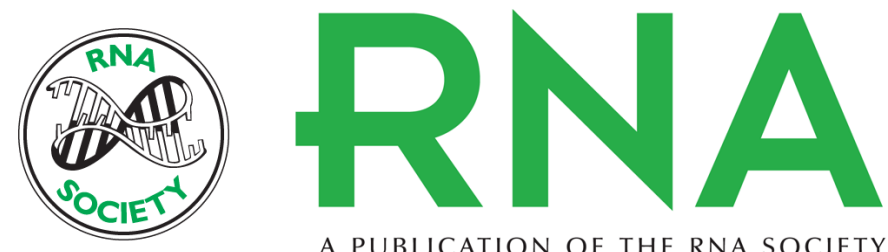

A PUBLICATION OF THE RNA SOCIETY

\section{Epistasis analysis of 16S rRNA ram mutations helps define the conformational dynamics of the ribosome that influence decoding}

Lanqing Ying and Kurt Fredrick

RNA 2016 22: 499-505 originally published online February 12, 2016

Access the most recent version at doi:10.1261/rna.054486.115

\section{References This article cites 45 articles, 21 of which can be accessed free at: http://rnajournal.cshlp.org/content/22/4/499.full.html\#ref-list-1 \\ Creative This article is distributed exclusively by the RNA Society for the first 12 months after the Commons full-issue publication date (see http://rnajournal.cshlp.org/site/misc/terms.xhtml). After 12 License months, it is available under a Creative Commons License (Attribution-NonCommercial 4.0 International), as described at http://creativecommons.org/licenses/by-nc/4.0/.}

Email Alerting Receive free email alerts when new articles cite this article - sign up in the box at the Service top right corner of the article or click here.

\section{|||||||| Providing Precise Solutions for your research.}

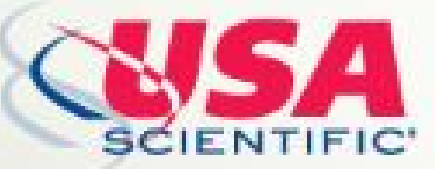

To subscribe to $R N A$ go to:

http://rnajournal.cshlp.org/subscriptions 